\title{
Application of the Butterworth's filter for excluding low-frequency trends
}

\author{
T.N. Dorokhova, N.I. Dorokhov \\ Astronomical Observatory, Odessa National University, \\ Shevchenko Park, Odessa 65014 Ukraine
}

\begin{abstract}
In the research of roAp stars low-frequency atmospheric and instrumental trends of data can be removed by the application of Butterworth's filter. Here we show the validity of such a technique by using a data set in which we combined the observed star's data and an artificial frequency spectrum. It is shown that the clearing of the data in the required frequency region is acquired due to the reduction of low-frequency noise, whereas the frequency solution practically does not change. The cleaning enables an easier detection of dominant frequencies in the investigated frequency region.
\end{abstract}

During some years we tested low-frequencies filters for reducing atmospheric and instrumental trends from observations of roAp stars obtained under nonideal photometric conditions. We examined such techniques by using artificial data series in the manner described by Breger (1990).

The programs PERIOD (Breger 1990), FOUR (Andronov 1994) and Period98 (Sperl 1998) were used for the analysis. The SPE package by Sergeev (1992) was initially applied for data reducing and filtering.

We prepared a data set consisting of time series measurements of a comparison star (10 $\left.0^{\text {th }} \mathrm{mag}\right)$ during three long nights (8-10 hours) and incorporated the artificial frequency spectrum with the parameters given in Table 1 . The resulting light curve is a combination of the observed atmospheric frequency distribution with an ideal frequency spectrum. The frequency spectrum slightly changed: the frequency at $112 \mathrm{c} / \mathrm{d}$ became $113 \mathrm{c} / \mathrm{d}(\mathrm{A} \sim 1 \mathrm{mmag})$ and at 81 $\mathrm{c} / \mathrm{d}$ a frequency appeared with $\mathrm{A} \sim 2 \mathrm{mmag}$.

The spectral window and amplitude Fourier spectrum of the combined pattern are presented in Fig. 1. 


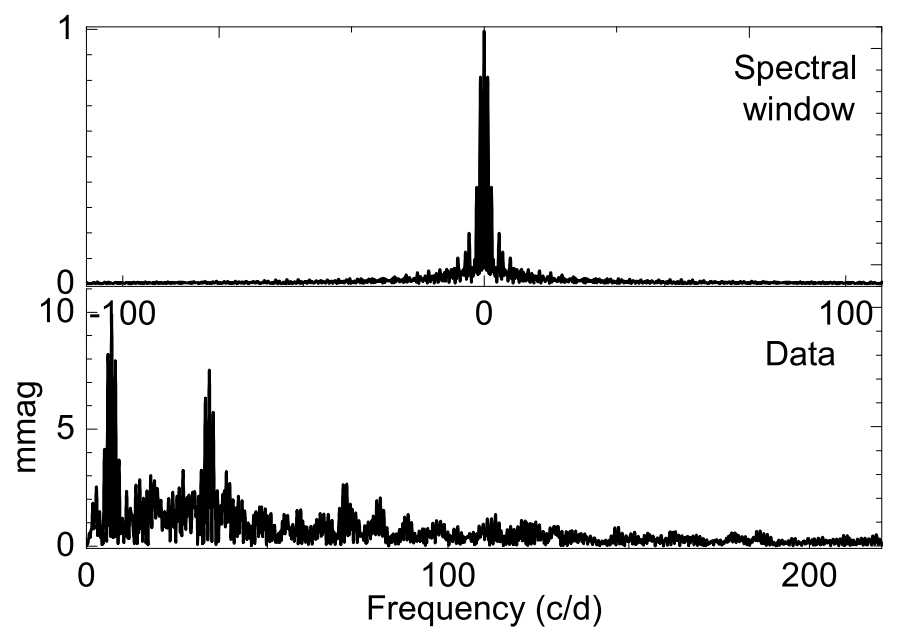

Figure 1: The spectral window and amplitude spectrum of the combined data set.

For the case of roAp stars the frequency region of interest ranges from 70 to $260 \mathrm{c} / \mathrm{d}$ Therefore, we are especially interested in the frequencies at 71,81 and $113 \mathrm{c} / \mathrm{d}$.

Due to the high noise level, $\mathrm{f}_{4}=113 \mathrm{c} / \mathrm{d}$ is undetectable and does not appear even after 12 steps of the analysis.

After that we applied the low-frequency Butterworth's filters of some different cutoff frequencies and removed the low-frequency trends of the data.

We define the low frequency Butterworth's filter in the following way:

$$
|H(f)|^{2} \approx(B / f)^{2 M} \quad \text { when } f \gg B
$$

Table 1: Artificial frequency spectrum.

\begin{tabular}{cccc}
\hline No & frq.(c/d) & Ampl & Phase \\
\hline 1 & 7 & 10 & 0.4 \\
2 & 34 & 7 & 0.1 \\
3 & 71 & 3 & 0.6 \\
4 & 112 & 1 & 0 \\
\hline
\end{tabular}




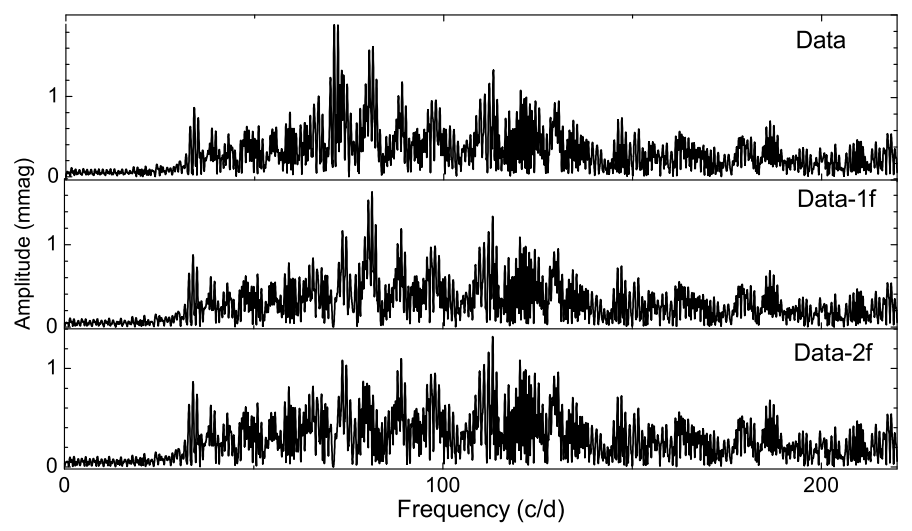

Figure 2: The amplitude spectrum of the data after removing the low-frequency trend by Butterworth's filter degree $M=2$ and cutoff frequency $B=5 \%$. The revealed frequencies are: $71.01 \pm 0.01 \mathrm{c} / \mathrm{d}(\mathrm{A}=1.9 \pm 0.13) ; 81.26 \pm 0.02 \mathrm{c} / \mathrm{d}(\mathrm{A}=1.6 \pm 0.1)$; $113.15 \pm 0.02 \mathrm{c} / \mathrm{d}(\mathrm{A}=1.3 \pm 0.1)$. The first low frequency peak is at $34 \mathrm{c} / \mathrm{d}$. Its amplitude has decreased by 8 times but the value of frequency is practically unchanged.

The filter with such parameters is close to ideal, i.e. passes only the required region of frequencies (see Otnes \& Enochson 1978).

From experience we selected the degree $\mathrm{M}=2$ and cutoff frequency $\mathrm{B}$ from 2 to $5 \%$. Here we present even more rigorous cutoff frequencies $B=5 \%$ (Fig. 2 ) and $B=7 \%$ (Fig.3) studying the filters' influence to the investigated frequency region.

As a result of filtering the low frequencies' amplitudes became lower (the frequencies are not changed). Figure 4 . shows the noise spectrum for different cutoff frequencies.

At $100 \mathrm{c} / \mathrm{d}$ the noise has been reduced by $20 \%$ for $B=5 \%$, by $35 \%$ for $\mathrm{B}=7 \%$ and up to $70 \%$ for $\mathrm{B}=10 \%$. The reduction of the noise level enables the astronomer to find the intrinsic frequencies sooner and more reliable.

These simple examples show that the frequency solution for the investigated region is not distorted even by adopting a rather rigorous filter. The application of low frequency filters appears to be more efficient and a better procedure than corrections with low degree polynomials. 


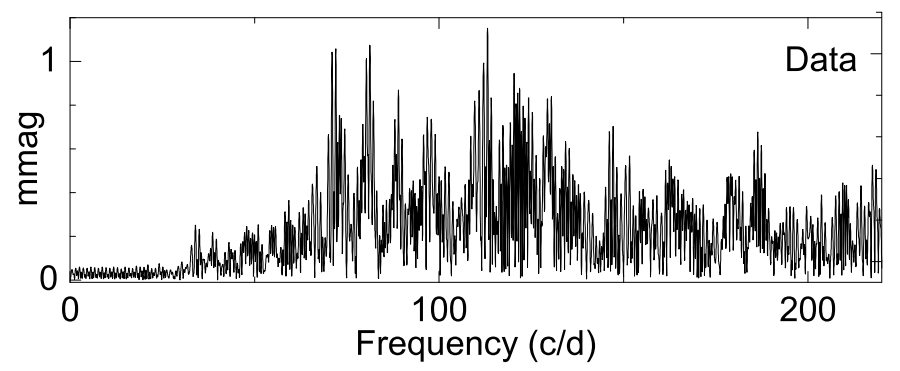

Figure 3: The amplitude spectrum of the data after removing the low-frequency trend by Butterworth's filter degree $M=2$ and cutoff frequency $B=7 \%$. The revealed frequencies are: $113.16 \pm 0.02 \mathrm{c} / \mathrm{d}(\mathrm{A}=1.1 \pm 0.1) ; 72.02 \pm 0.01 \mathrm{c} / \mathrm{d}(\mathrm{A}=1.06 \pm 0.1) ; 81.26$ $\pm 0.02 \mathrm{c} / \mathrm{d}(\mathrm{A}=1.0 \pm 0.1)$.

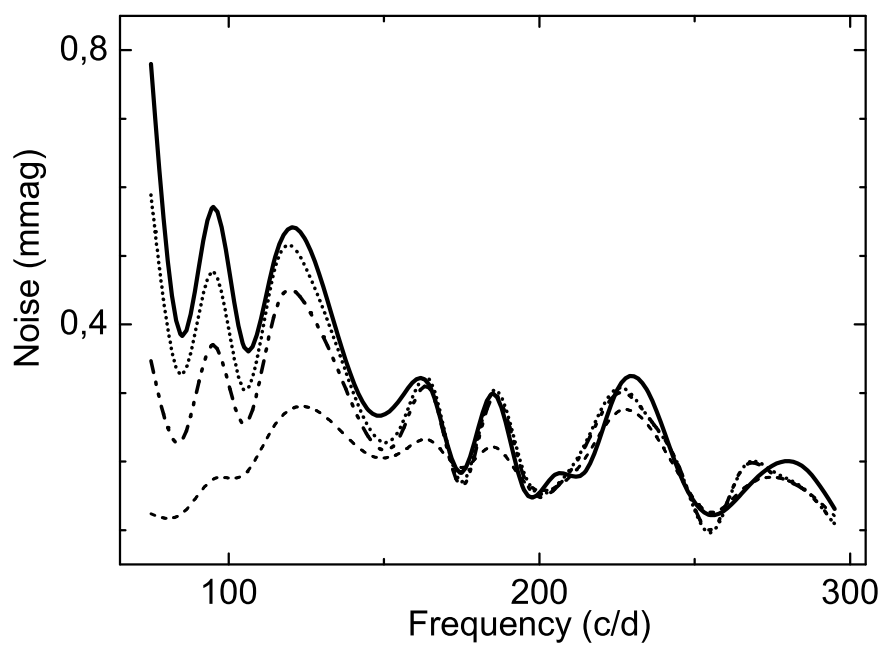

Figure 4: The noise spectrum for: solid - the unfiltered data; dotted - after the $M=2$ degree and $B=5 \%$ cutoff frequency filter; dash-dot-dot - after $B=7 \%$ cutoff frequency filter; dashed - after $B=10 \%$ cutoff frequency filter. 


\section{References}

Andronov I.L. 1994, Odessa Astronomical Publications, 7, 49

Breger M. 1990, CoAst, 20, 1

Otnes R. K., Enochson L. 1978, Applied time series analysis V.1. Basic Technologies, New York, p. 428

Sergeev 1992, private communication

Sperl 1998, CoAst, 111, 1 